\title{
Plurality and distributivity in Yaeyaman wh-questions *
}

\author{
Christopher Davis \\ University of the Ryukyus
}

\begin{abstract}
In Yaeyaman, a critically endangered Japonic language of the Southern Ryukyus, there is a distinction made between singular and plural wh-questions, with plurality indicated by reduplication of the indeterminate (wh) pronoun. I argue that reduplication of the indeterminate is triggered by a morpheme RED that requires the presence of non-atoms in the set of Hamblin alternatives denoted by its sister. When attached directly to an indeterminate pronoun, RED requires the presence of non-atomic, plural entities. I then show that reduplicated indeterminate subjects can be interpreted distributively in pair-list answers, while reduplicated indeterminate objects cannot. After showing that the distributive reading of the subject indeterminate cannot be modeled straightforwardly using a distributivizing operator attached to the VP, I suggest that it reflects morphological agreement between the subject indeterminate and a clause-level RED morpheme, which requires the existence of plural answers in the set of alternative propositions denoted by the question. The semantics of clause-level RED requires a distinction between atomic and plural answers that parallels the distinction between atomic and plural entities. I also compare the Yaeyaman data with reduplication in Korean questions, showing that the semantics of RED differs between the two languages.
\end{abstract}

Keywords: plurality, distributivity, indeterminates, wh-questions, pair-list answers, reduplication, Ryukyuan, Yaeyaman, Korean

\section{Introduction}

Yaeyaman is an endangered language of the Southern Ryukyus. Ryukyuan is a sister language group of Japanese, and shares its basic grammatical and typological properties. ${ }^{1}$ This paper focuses on data from the Taketomi dialect of Yaeyaman,

* I would like to thank first and foremost my primary language consultant for the data in this paper, Mr. Saburo Sakiyama, who patiently provided subtle judgments to an often frustrating deluge of questions about questions. Thanks to Eric McCready for discussion of the data, and Dorothy Ahn for help with the Korean data. I also thank the SALT 25 reviewers and audience members, as well as the members of the Semantics Reading Group in Japan, who gave valuable feedback and suggestions that have led to improvements in the paper. The research reported in this paper was supported in part by JSPS Grant in Aid for Research 24242014. All errors are my own.

1 For English-language information on Ryukyuan languages, see Uemura \& Lawrence (2003) for a descriptive overview of Ryukyuan, focused on Shuri Okinawan, Shimoji \& Pellard (2010) for 
traditionally spoken on the island of Taketomi. ${ }^{2}$ This dialect is critically endangered, and seems to no longer be regularly used for daily communication. The data reported here reflect the results of intensive interviews with a single speaker of the language; the small number of native speakers and their advanced age make extensive crossspeaker comparison difficult, but replication of the research with other speakers and other dialects of Yaeyaman is an ongoing project.

Yaeyaman wh-questions, like those in Japanese, are built using indeterminate pronouns (Kuroda 1965; Haspelmath 1997), which are also used in the formation of universal, existential, free-choice, NPI, and other constructions (Hagstrom 1998; Shimoyama 2001, 2006, 2011; Kratzer \& Shimoyama 2002; a.o.). In both (Tokyo) Japanese and Yaeyaman, indeterminates can be reduplicated, but the resulting interpretation is different in the two languages. ${ }^{3}$ Reduplicated indeterminates in Japanese are analyzed by Sudo (2013) as involving metalinguistic existential quantification. Cross-linguistically, reduplicated indeterminates are often interpreted as existential indefinites of some sort (Haspelmath 1997; Bylinina 2010).

Unlike Japanese, Yaeyaman exploits reduplication on its indeterminates to mark plurality in wh-questions, as illustrated by the following examples:

$$
\begin{aligned}
& \text { a. } \text { sinu=ya taa=du kitta-riyaa. } \\
& \text { yesterday=TOP who=FOC came-Q } \\
& \text { 'Who came yesterday?' } \\
& \text { b. } \text { sinu=ya taa+taa=du kitta-riyaa. } \\
& \text { yesterday=TOP who+who=FOC came-Q } \\
& \text { 'Who came yesterday?' }
\end{aligned}
$$

grammatical sketches of a number of Ryukyuan languages, and Heinrich, Miyara \& Shimoji (2015) for a large collection of papers on various topics in Ryukyuan linguistics. A large focus of research into Ryukyuan has been in relation to the phenomenon known as kakari musubi in Japanese, in which a "connecting" (kakari) particle "agrees" (musubi) with a predicate (see Shinzato \& Serafim (2013) for a recent synthesis of research into this phenomenon in Okinawan and Premodern Japanese, along with references to the very large research literature in this area). This phenomenon has attracted some attention in the formal literature as well, in particular as it is manifested in interrogative clauses in Okinawan (Sugahara 1996; Hagstrom 1998; Miyara 2007; Hiraiwa 2009). The role of focus particles in the semantics of interrogatives is not discussed in this paper, but see Davis (2014) for a discussion of the role they play in another variety of Yaeyaman. Integrating the semantic proposals in this paper with the semantics of kakari/focus particles is left for future research.

2 By far the largest reference work on Taketomi Yaeyaman is the Taketomi Dialect Dictionary (Maeara 2011), a large Taketomi to Japanese dictionary, including phonetic transcriptions and example sentences, as well as grammatical and cultural information.

3 As reported by Sudo (2013), there seem to be some dialects of Japanese in which reduplication of indeterminates leads to a plural wh interpretation, as in Yaeyaman. I am not aware of any research into such dialects. 
As descriptive labels, we can say that the non-reduplicated indeterminate in (1a) gives rise to a 'singular' wh-expression, while the reduplicated indeterminate in (1b) gives rise to a 'plural' wh-expression. ${ }^{4}$

There are a number of possibilities for how these descriptive labels can be spelled out semantically. In Section 2, I begin exploring these possibilities, adopting Hamblin's (1973) alternative semantics of questions, as developed for Japanese indeterminates by Kratzer \& Shimoyama (2002). This will be combined with a treatment of plurality based on Link 1983/2002. I show that several straightforward ways of spelling out the singular-plural indeterminate distinction within this basic framework do not work. I then argue indeterminates denote contextually restricted sets of alternatives, and that reduplication requires that this set contain at least some non-atomic entities. I then briefly turn to reduplicated indeterminates in Korean, showing that they seem to come with a stronger plural presupposition than those in Yaeyaman. In Section 3, I turn to multiple-wh questions, showing that reduplicated subject indeterminates can receive a kind of 'distributive' interpretation that is not available to object indeterminates. I suggest that this results from agreement with a higher operator that requires plural answers, rather than plural entities. I then briefly look at reduplicated multiple-wh questions in Korean, showing that they seem to differ semantically from those of Yaeyaman. Section 4 concludes.

\section{Singular and plural indeterminates}

\subsection{Alternatives and pluralities}

I begin by adopting the Hamblin-style alternative semantics of Japanese questions outlined in Kratzer \& Shimoyama 2002. ${ }^{5}$ Formally, denotations are 'raised' to sets of ordinary denotations; sentences thus denote sets of propositions. Declarative sentences denote singleton sets, while interrogatives/questions denote non-singleton sets. Sets of propositions are built up compositionally from pieces that themselves denote sets of ordinary meanings, which compose by pointwise function application. Japanese indeterminates (which play the role of wh-words in wh-questions) are taken

4 Like its sister language Japanese, Yaeyaman does not systematically mark plurality on its nominal expressions, although as in Japanese there are optional strategies for plural marking (see, for example, Nakanishi \& Tomioka's (2004) analysis of the plural suffix tachi in Japanese). In both Japanese and Yaeyaman, a non-productive process of reduplication can be used to mark plurality for a small set of nominal expressions; for example, Japanese yama 'mountain' has a reduplicated variant yamayama meaning 'mountains'. The connection between reduplication of nominals and that of indeterminates is suggestive, but I leave aside any investigation of the connection.

5 Hagstrom 1998 is as far as I am aware the first Hamblin-style treatment of Japanese sentences involving indeterminates, including wh-questions. Hagstrom's formal implementation is more complex than that in Kratzer \& Shimoyama 2002, and I stick here to the latter for the sake of simplicity. 
Plurality and distributivity in Yaeyaman wh-questions

by Kratzer \& Shimoyama to denote non-singleton sets of entities. For example, the Japanese indeterminate dare 'who' denotes the set of human entities: ${ }^{6}$

$$
\llbracket \text { dare } \rrbracket=\{x \mid \operatorname{human}(x)\}
$$

This denotation can then combine by pointwise function application with a predicate to form a set of alternative propositions:

$$
\begin{aligned}
& \llbracket \text { kita } \rrbracket=\{\lambda x . \text { came }(x)\} \\
& \begin{aligned}
\llbracket \text { dare kita } \rrbracket & =\{p \mid \exists x: \operatorname{human}(x) \wedge p=\operatorname{came}(x)\} \\
& =\{\operatorname{Mary} \text { came, Mariko came, Bill came, } \ldots\}
\end{aligned}
\end{aligned}
$$

Japanese does not exhibit a singular-plural distinction in its indeterminates. Kratzer \& Shimoyama do not discuss whether plural individuals are to be included in the denotation of dare, but the denotation in (2) gives a set of atomic alternatives. Two facts suggest that this set should be expanded to include non-atomic sums as well: the ability to felicitously answer such questions with a plural substitution for dare, and the ability to combine dare with collective predicates. First, consider the question in (5), and the possible answers in (5a) and (5b):

$$
\begin{aligned}
& \text { kinoo dare ga kita no? } \\
& \text { yesterday who NOM came Q } \\
& \text { 'Who came yesterday?' }
\end{aligned}
$$
a. Mariko ga kita yo.
Mariko NOM came SFP
'Mariko came.'

b. Mariko to Shizuko ga kita yo.

Mariko and Shizuko NOM came SFP

'Mariko and Shizuko came.'

Questions built from dare can be answered naturally with either singular (5a) or plural (5b) substitutions, suggesting that we need to include plural sums among the alternatives denoted by dare.

Indeterminates like dare can also be combined with collective verbs like atsumatta 'gathered'/'got together', which can only be predicated of plural individuals: ${ }^{7}$

a. dare ga atsumatta no?

who NOM gathered Q

'Who got together?'

6 World variables are suppressed throughout for convenience.

7 There seems to be some variation in judgments here, with some speakers not accepting (6a). 
b. * hanako ga atsumatta no?

Hanako NOM gathered Q

'Did Hanako get together?'

c. hanako to mariko ga atsumatta no?

Hanako and Mariko and gathered Q

'Did Hanako and Mariko get together?'

The contrast in grammaticality between (6b) and (6c) shows that the predicate atsumatta 'gathered' requires a plural subject.

Following Link (1983/2002), plural entities are modeled as sums of atomic entities; plural entities such as $e_{1} \oplus e_{2}$ are built out of the atomic entities $e_{1}$ and $e_{2}$ with the sum operator $\oplus$. Sums are structured by the part-of relation, $\leq$, and atoms are defined as those parts that have only themselves as parts. The domain of plural entities $* D$ is the closure of the domain of atomic entities $D$ under $\oplus$. Given the grammaticality of (6a) and the felicity of (5b), I conclude that the set of alternatives generated by Japanese dare 'who' contains among its alternatives sums of human entities (*humans) rather than just atomic entities. This requires the denotation in (2) to be revised as in (7).

$$
\begin{aligned}
& \llbracket \text { dare } \rrbracket=\{x \mid * \operatorname{human}(x)\}, \\
& \text { where } * \operatorname{human}(x)=1 \text { iff } \forall a:(a \leq x \& \operatorname{atom}(a)) \rightarrow \operatorname{human}(a)
\end{aligned}
$$

\subsection{Yaeyaman questions: some theoretical options}

Turning now to Yaeyaman, we find a contrast in acceptability between singular and plural indeterminates in sentences with the predicate atsõoritta 'gathered'/'got together', as illustrated below:

$$
\begin{aligned}
& \text { a. ?? taa=du atsõõritta-rijaa. } \\
& \text { who=FOC gathered-Q } \\
& \text { 'Who got together?' } \\
& \text { b. taa+taa=du atsõõritta-rijaa. } \\
& \text { who+who=FOC gathered-Q } \\
& \text { 'Who got together?' }
\end{aligned}
$$

While sentence $(8 b)$ with a reduplicated subject indeterminate is perfectly acceptable, the same sentence in (8a) without reduplication is judged to be worse. What then is the degree of and reason for the unacceptability of (8a)?

Given the inclusive semantics of the Japanese indeterminate dare argued for above, there are several obvious possibilities for distinguishing Yaeyaman singular 
and plural indeterminates. The non-reduplicated indeterminate taa 'who' might be a strong singular (following loosely the terminology in Sauerland, Anderssen \& Yatsushiro 2005), denoting only atoms as in (9a), or a weak singular, denoting both atoms and sums as in (9b) (note that D is the set of all atomic entities). Similarly, the reduplicated plural might be a strong plural, denoting only non-atomic sums as in $(9 \mathrm{c})$, or a weak plural, denoting both atoms and non-atoms as in (9d).

$$
\begin{array}{lll}
\text { a. } \llbracket t a a_{s} \rrbracket & =\{x \mid * \operatorname{human}(x)\} \cap \mathrm{D} & =\{a, b, c, \ldots \\
\text { b. } \llbracket t a a_{w} \rrbracket & =\{x \mid * \operatorname{human}(x)\} & =\{a, b, c, \ldots, a \oplus b, a \oplus c, \ldots\} \\
\text { c. } \llbracket t a a+t a a_{s} \rrbracket & =\{x \mid * \operatorname{human}(x)\} \backslash \mathrm{D} & =\{ \\
\text { d. } \llbracket t a a+t a a_{w} \rrbracket & =\{x \mid * \operatorname{human}(x)\} & =\{a, b, c, \ldots, a \oplus b, a \oplus c, \ldots\}
\end{array}
$$

The possibilities in (9) can be put together in four ways. First, there are two possible strong singular theories. Given that the singular is strong (9a), it could be contrasted with either a strong plural $(9 \mathrm{c})$, or with a weak plural (9d). Either version of the strong singular theory would explain the unacceptability of (8a) by appealing to the fact that all of the alternative propositions built from a strong singular indeterminate and a collective predicate are semantically anomalous, leading to a question denotation with no semantically non-anomalous answers. A strong plural would, in contrast, give only non-anomalous alternatives, while a weak plural would at least provide some non-anomalous alternatives along with the anomalous ones produced by the strong singular. In either case, the theory would rule out ( $8 \mathrm{a})$ by saying that it simply fails to provide any coherent alternatives.

If we instead adopt a weak theory of the singular form, as in (9b), then only the strong plural denotation in (9c) will provide a different set of alternatives from the singular. Under this theory (weak singular, strong plural), we would say that although the unreduplicated form in (8a) does provide coherent alternatives in addition to incoherent ones, its use is dispreferred in favor of the plural form in (8b), which provides all the coherent alternatives of (8a) without any of the incoherent ones.

As a final alternative, we could adopt an approach combining the weak singular in (9b) with the weak plural in (9d). This combination would fail to distinguish the singular and plural forms in terms of the alternatives denoted, and the semantic contrast would need to be supplied in some other way.

\subsection{A problem}

With the most obvious theoretical options on the table, I now turn to facts showing that none of these options can be correct. To do so, consider the felicity of the questions in (1) in each of three contexts. In the first context, the speaker assumes that only a single person came. Call this the 'singular answer assumption' context. In the second context, the speaker assumes instead that multiple people came. Call 
this the 'plural answer assumption' context. Finally, in the third context, the speaker has no prior assumptions about how many people came; call this the 'no assumption' context. The acceptability of singular (1a) and plural (1b) questions in these contexts is summarized in (10).

\begin{tabular}{lll} 
& $(1 \mathrm{a})$ & $(1 \mathrm{~b})$ \\
\hline Context 1: Singular Answer Assumption & OK & BAD \\
Context 2: Plural Answer Assumption & OK(?) & OK \\
Context 3: No Assumption & OK & OK
\end{tabular}

Looking first at the plural question in (1b), we see that reduplication is acceptable both when the speaker assumes that the answer will be plural, as well as when the speaker makes no assumptions at all. It is only bad when the speaker has a strong prior assumption that the answer will be singular. From this, it seems that the reduplicated plural must be weak, including both atomic and non-atomic individuals in the alternatives it denotes, as in (9d). If it were strong, as in (9c), then it would only generate plural answers, taking singular answers off the table altogether. This would in turn lead us to expect that the plural question in (1b) is a marked option in context 3 , contrary to fact.

Sentence (1a), with a non-reduplicated singular wh-subject, is compatible and perfectly natural with the first and third contexts. In the second context, with a plural answer assumption, (1a) is not as natural as (1b). It does not, however, seem to be ruled out completely. Instead, my consultant reports that while (1b) is in some sense 'better' or 'more precise', (1a) is not entirely impossible. In any case, the felicity of (1a) in context 3 forces us to conclude that the singular is weak as well, since if it were strong, we would predict it to take singular answers off the table completely, and thus be a marked option in both contexts 2 and 3 .

The felicity pattern in (10) leads us to the undesirable conclusion that both singular and plural indeterminates are weak in Yaeyaman, given that they are both equally felicitous in context 3 . But then both forms would denote the same set of alternatives, and the contrast in contexts 1 and 2 of (10), as well as the contrast in (8), would be left unexplained. There is something not quite right with our formulation of the theoretical options then. In the next section, I show how this problem can be handled by having indeterminates denote only a contextually salient subset of the alternatives described in this section; this, coupled with a presupposition triggered by reduplication that the contextually determined subset of alternatives include at least some non-atomic alternatives, can account for the data in this section. 
Plurality and distributivity in Yaeyaman wh-questions

\subsection{A solution}

I propose that indeterminates denote a set of alternatives whose membership is determined by a contextual variable $\mathrm{C}$. The membership of $\mathrm{C}$ can be restricted by presupposition. Yaeyaman taa 'who' comes with a presupposition that the elements of $\mathrm{C}$ are (sums whose atoms are) human, as in (11). Other indeterminates will come with their own presuppositions; for example, noo 'what' comes with a presupposition that the elements of $\mathrm{C}$ are (sums whose atoms are) inanimate. And so on for other indeterminates.

$$
\begin{aligned}
& \llbracket t a a \rrbracket=\mathrm{C} \\
& \text { presupposition: } \mathrm{C} \subseteq\left\{\mathrm{x} \mid \mathrm{x} \in *^{*} \operatorname{human}(\mathrm{x})\right\}
\end{aligned}
$$

Reduplication is triggered by a morpheme RED, which attaches to an indeterminate, and has the denotation in (12).

$$
\llbracket \operatorname{RED} \alpha \rrbracket=\llbracket \alpha \rrbracket \text { if } \llbracket \alpha \rrbracket \nsubseteq \text { ATOMS, else undefined. }
$$

The reduplicated indeterminate taa+taa results from the combination of RED with taa, giving the semantics in (13).

$$
\begin{aligned}
& \llbracket \text { RED taa } \rrbracket=\mathrm{C} \\
& \text { presuppositions: } \mathrm{C} \subseteq\{\mathrm{x} \mid \mathrm{x} \in \text { *human( } \mathrm{x})\} \text { and } \mathrm{C} \nsubseteq \text { ATOMS }
\end{aligned}
$$

Reduplication adds the presupposition that C not contain only atoms; in other words, the reduplicated form presupposes that the alternatives contain at least some nonatomic sums. It says nothing about whether $\mathrm{C}$ also contains atoms. In effect, reduplication creates an 'anti-strong-singular' form; it is compatible with both weak and strong plural sets of alternatives (sets containing at least some or potentially only non-atomic sums), but is incompatible with strongly singular alternative sets (those containing only atoms).

The denotations in (11) and (13) help explain the felicity pattern in (10) as follows. First, consider what $\mathrm{C}$ would look like in the three contexts in (10):

$$
\begin{aligned}
& \mathrm{C}_{1}=\{\mathrm{a}, \mathrm{b}, \mathrm{c}, \ldots, \quad\} \quad \text { (singular assumption) } \\
& \mathrm{C}_{2}=\{\mathrm{a} \oplus \mathrm{b}, \mathrm{a} \oplus \mathrm{c}, \ldots\} \quad \text { (plural assumption) } \\
& \mathrm{C}_{3}=\{\mathrm{a}, \mathrm{b}, \mathrm{c}, \ldots, \mathrm{a} \oplus \mathrm{b}, \mathrm{a} \oplus \mathrm{c}, \ldots\} \quad \text { (no assumption) }
\end{aligned}
$$

$\mathrm{C}_{1}$ is incompatible with the presuppositions of plural taa+taa, correctly predicting that only the singular can be used in context $1 . \mathrm{C}_{3}$ is compatible with the presuppositions of taa+taa, as well as those of taa, correctly predicting that both forms are possible in context 3 . This leaves $\mathrm{C}_{2}$, where only non-atomic sums are considered as possible alternatives. The presuppositions of both taa+taa and taa are satisfied by this set, and while both are possible, it seems that the plural is preferred. This 
preference becomes much stronger when it is the semantics of the predicate, rather than the context, that rules out atomic alternatives, as shown by the contrast in (8).

The theory as it stands is thus too liberal in its predictions, failing to explain the preference for reduplicated forms in plural-assumption contexts, and in sentences with collective predicates. Although our theory predicts that taa can denote a $\mathrm{C}$ with only non-atomic sums, in accordance with the assumptions in context 2 , this seems to be a marked move, given the availability of taa+taa, which explicitly registers the fact that non-atoms are to be considered. Something similar will need to be said for the apparently much stronger contrast between taa and taa+taa seen in (8), where a predicate that is semantically incompatible with atoms seems to demand a plural indeterminate argument.

The slight contrast in context 2 of (10), and the significant contrast in (8), might be taken to result from a principle like Maximize Presupposition (Heim 1991, Sauerland et al. 2005), which requires that if two forms differ only in that one has stronger presuppositions, the form with the stronger presuppositions should be used in contexts where those presuppositions are satisfied. This principle says that, given two competing forms, the one with the stronger presuppositions must be used where possible. Given the analysis above, in Yaeyaman the plural has stronger presuppositions than the singular, and the principle thus predicts that the plural should be used wherever its presuppositions are licensed.

While this would make sense of the contrast in context 2 of (10), it makes unwanted predictions as well. In particular, it predicts that the singular should be equally infelicitous in context 3 , since the stronger presuppositions of the plural are licensed there. Given that there is no assumption about the plurality of the answer, the speaker of course considers a plural answer possible, and so the plural form is licensed. Maximize Presupposition should thus force the use of the reduplicated indeterminate in context 3 , contrary to fact.

I suggest that the contrast results instead from a conversational implicature associated with the singular form, to the effect that $\mathrm{C}$ contains atoms. This implicature is the mirror image of the presupposition encoded by reduplication, which requires the presence of non-atoms. This implicature is violated in context 2 in (10), where the context eliminates singular substitutions from consideration. It is also violated in (8), where the semantics of the collective predicate is incompatible with the atomic alternatives demanded by the conversational implicature of the singular form.

In order to explain the difference in the degree of the contrast in (8) and context 2 of (10), consider what happens when we change the context in order to satisfy the implicature of the singular form. In the case of (8), the implicature forces the inclusion of atomic alternatives, which leads to semantically anomalous alternatives in which the collective predicate is fed atomic arguments. Satisfying the implicature associated with the singular form forces us to consider semantically ill-formed 
alternatives, which results in a strong preference for the plural form.

By contrast, consider what happens if we force the inclusion of atomic alternatives in context 2 of (10). In particular, imagine we do so by including the atoms from which the non-atomic sums in the original set are composed. This does no violence to the semantic coherence of any alternatives. First, the predicate in this sentence distributes to the atoms of any non-atomic argument; so, for example, if it is true that $a \oplus b$ came, then it follows both that $a$ came and that $b$ came. Second, the alternative propositions generated by the Hamblin semantics adopted in this paper are non-exhaustive and independent. That is, the truth of a particular alternative proposition is in general compatible with the truth of any other alternative (in fact, the truth or falsity of any alternative proposition is in general independent of that of any other alternative). ${ }^{8}$ Thus, by including atoms in $\mathrm{C}$, all that happens is the generation of weaker alternative answers, all of which are entailed by the stronger answers that would have been generated on the basis of a $\mathrm{C}$ containing only non-atoms in any case. While unnecessary in a context that assumes a plural answer, there is no real damage done, and hence the dispreference for the singular form here is relatively weak.

Finally, consider what happens if we use the plural form in context 1 of (10), in which it is assumed that the answer will be singular. Here, the presupposition of the plural indeterminate would force us to include non-atoms in C. This in turn would generate alternative propositions such as ' $a \oplus b$ came'. The truth of such alternatives is ruled out by the prior assumptions made in this context (namely, that only one person came). Use of the plural form thus forces us to include propositions which are assumed to be false. Given a pragmatic principle to the effect that alternatives known to be false should not be included in the set denoted by a question, this move becomes highly marked, and the use of the plural form is ruled out in this context.

\subsection{Summary and crosslinguistic comparison}

The upshot of the above discussion is that both singular and plural indeterminates can in principle denote sets that contain both atoms and sums. This explains the fact that both forms are possible in context 3 of (10), where no assumptions are made about the plurality of the answer. Plural forms are restricted in that they cannot denote sets containing only atoms, and this explains the fact that they are not allowed in singular assumption contexts. Finally, singular forms are associated with an implicature that they denote sets containing at least some atoms, explaining the preference for plural forms in plural assumption contexts and in sentences with collective predicates. The account contrasts with the presuppositional semantics of number in Sauerland

8 This is a feature of Hamblin semantics that contrasts with the partition semantics of Groenendijk \& Stokhof (1984) and others, in which possible answers are mutually incompatible. 
et al. 2005, where singular forms have a strong singular presupposition, and plural forms are weak, lacking any presupposition at all. The account squares with Horn's 'division of pragmatic labor', where marked forms encode marked meanings, and bears an affinity to the proposal in Farkas \& de Swart 2010, who argue for a weak singular / strong plural theory of number marking in English.

Chung (1999) and Kim (1999) report a distinction in Korean between nonreduplicated indeterminates (what they call wh-singlets) and reduplicated indeterminates (what they call wh-doublets). As in Yaeyaman, the reduplicated Korean forms give rise to a plural interpretation. According to Chung, non-reduplicated forms are compatible with both singular and plural answers. This is also true of Yaeyaman. The reduplicated form, however, is reported not to license a singular answer, as illustrated by the contrasting felicity of the answers in (15). This contrasts with the judgments reported here for Yaeyaman, where singular and plural answers are equally felicitous responses to a question with a reduplicated indeterminate.

$$
\begin{aligned}
& \text { A: ecey nwukwu-nwukwu o-ess-ni? } \\
& \text { yesterday who-who-NOM come-PST-QE } \\
& \text { 'Who (Pl) came yesterday?' } \\
& \text { B: \# John-i o-ess-ta. } \\
& \text { John-NOM come-PST-DE } \\
& \text { 'John did.' } \\
& \text { B': John-kwa Mary-ka o-ess-ta. } \\
& \text { John-and Mary-NOM come-PST-DE } \\
& \text { 'John and Mary did.' }
\end{aligned}
$$

The Korean facts can be accounted for by modifying the presupposition associated with reduplication. In Yaeyaman, we have a weak presupposition, requiring only that some alternatives in the contextually specified set be non-atomic, as defined in (13). In Korean, it seems that reduplication comes with a stronger presupposition, requiring that all alternatives be non-atomic, as in (16):

$$
\begin{aligned}
& \llbracket \operatorname{RED} \alpha \rrbracket=\llbracket \alpha \rrbracket \text { if } \llbracket \alpha \rrbracket \cap \text { ATOMS }=\emptyset, \text { else undefined. } \\
& \llbracket n w u k w u+n w u k w u \rrbracket=\mathrm{C} \\
& \text { presupposition: } \mathrm{C} \subseteq\{x \mid \text { *human }(x)\} \text { and } \mathrm{C} \cap \text { ATOMS }=\emptyset
\end{aligned}
$$

In the rest of the paper, I consider the semantics of multiple-wh questions in Yaeyaman, and show that distributive interpretations are available for plural subject indeterminates, but not for plural object indeterminates. I argue that this interpretation results when RED attaches at a clausal level, requiring that the set of propositions denoted include non-atoms, which in the case of multiple-wh questions correspond to pair-list answers. 
Plurality and distributivity in Yaeyaman wh-questions

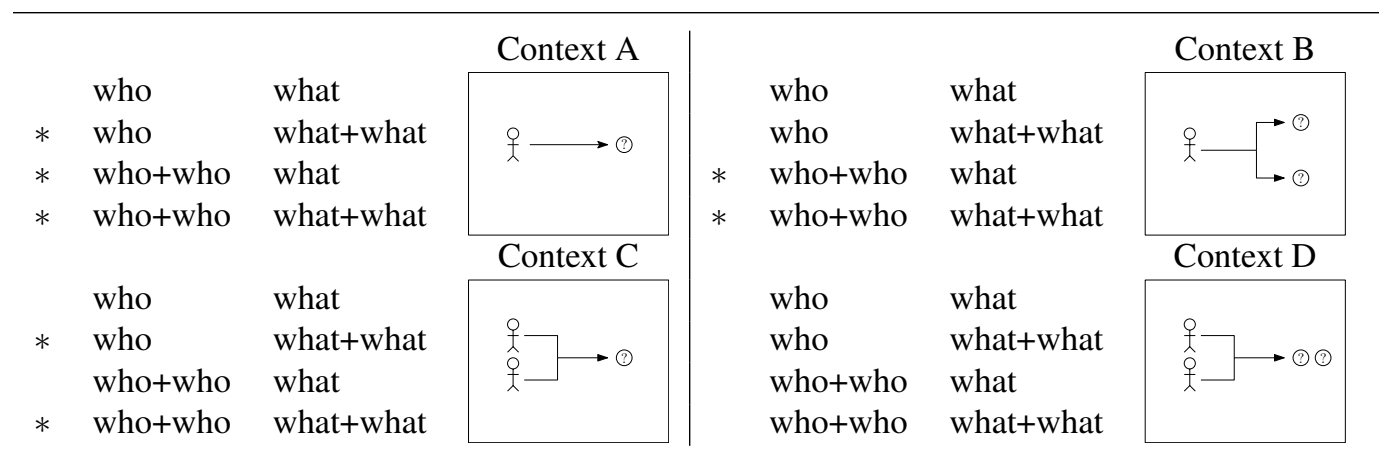

Table 1 Single pair answer contexts

\section{Multiple wh-questions and distributivity}

\subsection{Basic data}

The sentence in (18) is a multiple-wh question, with indeterminates in both subject and object position.

taa $(+$ taa $)=d u \quad$ noo $(+$ noo $)=y u \quad$ mucikee-riyaa.

who $(+$ who $)=$ FOC what $(+$ what $)=$ ACC brought $-Q$

'Who brought what?'

This sentence has four variants, depending on whether either or both of the subject and object indeterminates are reduplicated. The difference in meaning between these four possibilities is made clear by their acceptability in each of the four contexts illustrated in Table 1. The contexts are represented by pictures indicating the number of bringers and the number of things brought. Judgments were collected by presenting such pictures to my language consultant, and asking for the felicity of the four variants of (18) in each context. Lines connecting the bringers and things brought indicate the fact that each of these contexts were ones in which a single pair answer was expected (pair-list contexts are considered just below). The acceptability of the four variants is summarized in Table 1 (a star $*$ represents unacceptability in the given context). For simplicity, I ignore the (rather weak) implicature associated with singular forms in what follows; judgments of slight infelicity deriving from this implicature are thus ignored in the data below.

Context A requires alternative propositions of the form $B(x, y)$, where $x$ and $y$ are atoms. Reduplication of the subject or object indeterminate presupposes non-atomic alternatives for $x$ and $y$, respectively, and thus is ruled out in this context, since such alternatives are not taken as possible answers. Context $B$ requires alternatives of the form $B\left(x, y_{1} \oplus y_{2}\right)$, with atomic subjects and non-atomic objects. This licenses reduplication of the object indeterminate but not the subject indeterminate. Context 


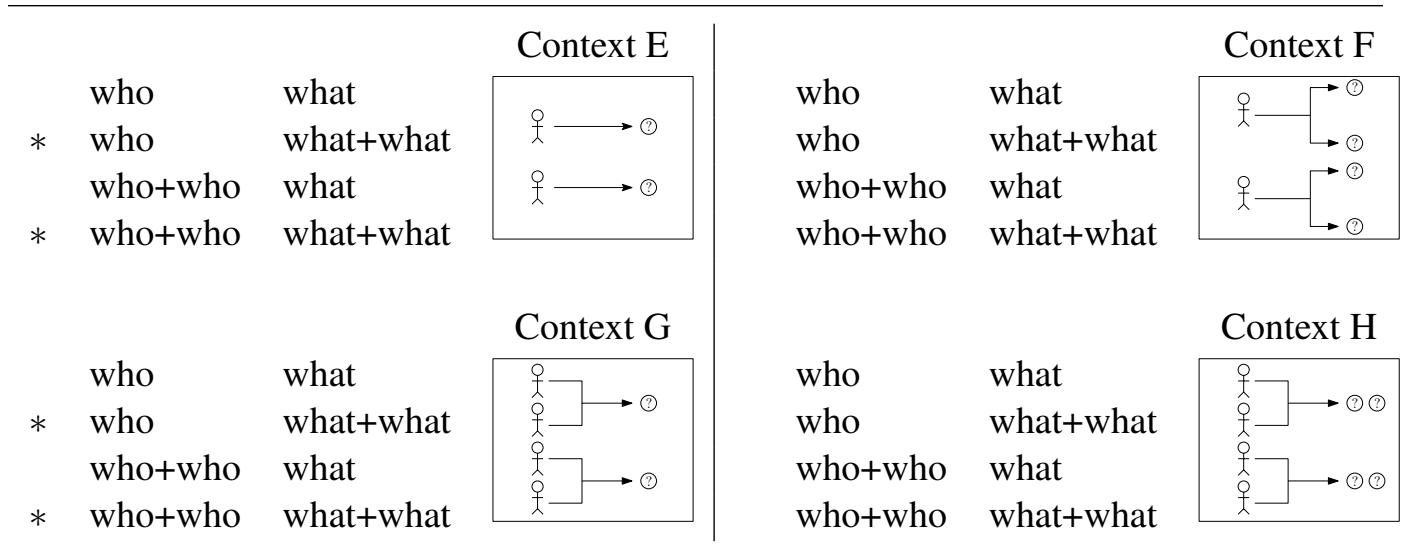

Table 2 Pair-list answer contexts

$\mathrm{C}$ is the reverse situation, in which alternative propositions must be of the form $B\left(x_{1} \oplus x_{2}, y\right)$. This licenses a reduplicated subject, but not an object. Context D requires answers of the form $B\left(x_{1} \oplus x_{2}, y_{1} \oplus y_{2}\right)$, licensing reduplication of both the subject and object indeterminates.

These facts follow straightforwardly from the semantics presented in the last section. Both subject and object indeterminates introduce contextually-determined alternative sets of individuals. These sets can in principle contain atoms only, nonatomic sums only, or a mix of the two. Reduplication in either position blocks the possibility of only atomic alternatives in that position, and thus requires that answers with non-atomic entities in that position be live possibilities in the context of utterance.

Turning now to contexts requiring a pair-list answer, we find a contrast between reduplicated subject and object indeterminates, illustrated by the felicity patterns for the contexts in Table 2. The upper contexts in Table 2 represent situations where a pair-list answer is expected, and in which there is an atomic subject in each pair. In other words, there is a plurality of subjects across pairs, but within each pair the subject is atomic. The bottom contexts, by contrast, have a plural subject within each pair. In all of these contexts, questions with a reduplicated subject indeterminate are acceptable. It thus seems that plural subject indeterminates are compatible with a kind of 'distributive' interpretation, since otherwise we would require a plural subject within (at least some) alternatives. Turning to the objects in these contexts, we find a contrast with the pattern for subjects described above. While each context has a plurality of objects, in the left contexts these are distributed over the pairs, so that each pair has an atomic object. In such contexts, reduplication of the object indeterminate is unacceptable. Only in contexts like those on the right, in which there is a plurality of objects within pairs, can the reduplicated object be used. This 
Plurality and distributivity in Yaeyaman wh-questions

contrast points to a difference in the availability of what we might think of as a 'distributive' reading for plural indeterminates in subject versus object position. In subject position, the plural indeterminate can distribute over pairs, while in object position it cannot.

\subsection{Trying to get distributive interpretations with a distributivity operator}

A first approach to analyzing the contrast in the availability of distributive readings of plural subject and object indeterminates would be to posit a distributivity operator that allows for distribution over subjects, but not over objects. Candidates for such an operator are not hard to find in the literature. As one example, consider the distributive operator $D$, as formalized in Lasersohn 1998:

(19) For any (one-place) predicate $P$ and sum of individuals $x:{ }^{D} P$ holds of $x$ iff $P$ holds of each individual part of $x$.

Lasersohn discusses a widely remarked-upon problem for this kind of analysis of distributivity, namely that it seems to predict that only subjects should allow for a distributive interpretation, since it is defined only for one-place predicates, and thus cannot apply to a transitive verb which has not yet taken an object argument. Lasersohn (1998) seeks to remedy the situation by generalizing the semantics of the distributive operator to all $n$-place predicates, while Roberts (1987) handles distribution over objects by lambda-abstracting over the object position of a saturated predicate, deriving a one-place predicate which can yield a distributive reading for plural objects.

Since with Yaeyaman indeterminates, distribution over pairs is only possible for subjects, perhaps this 'defect' of such distributivity operators can explain the asymmetry. While tempting, this analysis does not give the correct results. Assume for concreteness that the set of alternative objects is $\mathrm{C}_{o}=\{e, f, g\}$. Then, the VP of the question will denote the set of alternative properties $\left\{P \mid \exists y \in \mathrm{C}_{o}: P=\lambda x . B(x, y)\right\}$. Now, assume that the set of alternative subjects is $\mathrm{C}_{s}=\{a \oplus b, a \oplus c, b \oplus c\}$. Applying the denotation of the VP to the denotation of the subject would result in the following set of alternative propositions:

$$
\begin{aligned}
\llbracket \text { who+who [brought what }] \rrbracket & =\left\{p \mid \exists x \in \mathrm{C}_{s}, y \in \mathrm{C}_{o}: p=B(x, y)\right\} \\
& =\{B(a \oplus b, e), B(a \oplus c, e), B(b \oplus c, e), \ldots\}
\end{aligned}
$$

While this set will work for contexts $\mathrm{G}$ and $\mathrm{C}$, it won't do for context E, in which we need to 'distribute' the plural alternatives in $\mathrm{C}_{s}$ over pairs. Let's try to do this by applying the distributive operator $D$ :

$$
\begin{aligned}
& \left.\llbracket \text { who+who }{ }^{D} \text { [brought what }\right] \rrbracket=\left\{p \mid \exists X \in \mathrm{C}_{s} \forall x \in X: \exists y \in \mathrm{C}_{o}: p=B(x, y)\right\} \\
& =\{B(a, e) \wedge B(b, e), B(a, e) \wedge B(c, e), B(b, e) \wedge B(c, e), \ldots\}
\end{aligned}
$$


This is not the result we are after. What we need is a way to let the atoms of a plural subject distribute over different alternatives from the set denoted by the object indeterminate. The distributivity operator does not do this; it only allows us to distribute plural subjects over the same object.

\subsection{Plural answers}

In what follows, I suggest an analysis under which the 'distributive' interpretation of plural indeterminate subjects results from agreement with a clause-level RED that requires the existence of plural answers among the set of possible answers denoted by the question. To see how this approach works, first recall that the alternative propositions derived by our semantics are non-exhaustive and independent, as is generally true in a Hamblin-style alternative semantics. As discussed by Lahiri (1991, 2002) and Chierchia (1993), these alternatives can form the basis for a fuller set of possible propositions $Q^{\prime}$ by generating the set of all conjunctions of subsets of the original set of propositions, as defined below (note that set intersection is used here for conjunction, given that propositions are sets of possible worlds):

$$
\begin{aligned}
& \text { Ans }(p, Q) \text { if and only if } \exists S \in \mathscr{P}(Q)[p=\cap S] \\
& Q^{\prime}=\{p \mid \text { Ans }(p, Q)\}
\end{aligned}
$$

(cf. Lahiri 2002: 69-70)

For example, given a set of three Hamblin alternative propositions (which are in general logically independent, non-exhaustive alternatives):

$$
\begin{aligned}
Q & =\left\{p_{1}, p_{2}, p_{3}\right\} \\
Q^{\prime} & =\left\{p_{1}, p_{2}, p_{3}, p_{1} \cap p_{2}, p_{1} \cap p_{3}, p_{2} \cap p_{3}, p_{1} \cap p_{2} \cap p_{3}\right\}
\end{aligned}
$$

Lahiri (2002: 70-73) shows that $Q^{\prime}$ defines a Boolean algebra, whose atoms (24a) are the original propositions in $Q$, and whose non-atoms (24b) are conjunctions of non-singleton, non-empty subsets of the set of atoms:

$$
\begin{aligned}
& \text { a. } \operatorname{ATOMS}_{Q^{\prime}}=\left\{p_{1}, p_{2}, p_{3}\right\}=Q \\
& \text { b. } Q^{\prime}-\operatorname{ATOMS}_{Q^{\prime}}=\left\{p_{1} \cap p_{2}, p_{1} \cap p_{3}, p_{2} \cap p_{3}, p_{1} \cap p_{2} \cap p_{3}\right\}
\end{aligned}
$$

Formally, the structure is parallel to the one used by Link (1983/2002) to model plural entities.

In a multiple-wh question whose Hamblin denotation is $Q$, a single-pair answer corresponds to an atomic proposition of $Q^{\prime}$, i.e., to a proposition from the original set $Q$. A pair-list answer can be thought of as a non-atomic proposition from the expanded set $Q^{\prime}$; that is, pair-list answers are in effect plural answers. Given the formal parallel between the algebra of answers and that of entities, I suggest that reduplication of (subject) indeterminates can be interpreted as providing a 
Plurality and distributivity in Yaeyaman wh-questions

presupposition about the plurality of possible answers to the question; namely, that the speaker considers it possible that a non-atomic answer (here, a pair-list answer) is a true and relevant answer to the question.

Space limitations prevent me from developing the proposal in sufficient detail, but in the rest of this section I sketch one way that this idea might be spelled out. First, I suggest that there is a pluralizing operator $*$ that operates on the set of (atomic) Hamblin alternatives $Q$ to generate the expanded set $Q^{\prime}$ :

$$
\llbracket * Q \rrbracket=Q^{\prime}
$$

I assume that the $*$ operator is freely available, optional, and phonologically null. Thus, a multiple-wh question with non-reduplicated indeterminates is in principle ambiguous between a single pair interpretation (no $*$ operator) and a pair-list interpretation (generated by the $*$ operator).

Given the algebraic structure of $Q^{\prime}$, I suggest that RED can attach at the clause level, above $*$, where it generates the presupposition that its complement (here, a set of propositions) contain at least some non-atoms; the denotation of RED is repeated below, with atomicity relativized to $Q^{\prime}$ :

$$
\llbracket \operatorname{RED} \alpha \rrbracket=\llbracket \alpha \rrbracket \text { if } \llbracket \alpha \rrbracket \nsubseteq \text { ATOMS }_{Q^{\prime}} \text {, else undefined. }
$$

By attaching RED at the clause level, we generate a requirement that the set of propositions contain non-atoms from $Q^{\prime}$. This is only possible if we have pluralized the question by attaching $*$ first; the original Hamblin set $Q$ has only atomic propositions. Attaching RED at the CP level thus forces pluralization of the question with $*$, and requires the existence of plural (here, pair-list) answers in the set of alternatives.

Morphologically, RED is spelled out by reduplicating the structurally closest indeterminate. This accounts for the subject-object asymmetry described above. Under this analysis, the 'distributive' reading of reduplicated subject indeterminates in fact results from a higher-level operator that generates a presupposition about the existence of plural answers; the fact that such readings are limited to reduplicated subjects follows from locality in the morphological spellout of RED, which triggers agreement on the closest available indeterminate. ${ }^{9}$

9 The subject-object asymmetry seen with plural indeterminates is reminiscent of the asymmetry in the availability of distributive interpretations of quantifiers appearing in wh-questions (May 1985), as illustrated in pairs like the following:

(i) a. Who ${ }_{i}$ does everyone like $\mathrm{t}_{i}$ ?

b. $\mathrm{Who}_{i}$ likes everyone?

(Chierchia 1993: 181)

Both sentences allow for a singular constituent answer, like that in (0a), while only (0a) allows for a list answer, like that in $(0 b)$ :

(ii) a. Singular Constituent Answer: Professor Smith 
Davis

\subsection{Multiple plural wh and pair list answers in Korean}

According to Kim (1999), the following Korean sentence, in which both object and indirect object indeterminates are reduplicated, only allows for a pair-list interpretation:

$$
\begin{aligned}
& \text { Mary-ka nwukwu-nwukwu-eykey mwues-mwues-ul cwuess-ni? } \\
& \text { Mary-NOM who-who-tor what-what-ACC gave-Q }
\end{aligned}
$$

'Who did Mary give what?'

(Kim 1999: 392)

Kim does not provide examples of acceptable and unacceptable answers, but presumably the restriction to pair-list answers would rule out the use of (27) in all the contexts in Table 1. Dorothy Ahn (pc) confirms Kim's intuition that this question requires a pair-list answer, and suggests the following as a natural answer:

$$
\begin{aligned}
& \text { John-eykey chocolate-ul cwu-ko Bill-eykey satang-ul cwuesse. } \\
& \text { John-to chocolate-ACC give-CONJ Bill-to candy-ACC gave }
\end{aligned}
$$

'(She) gave chocolate to John and candy to Bill.'

The example in (27) has two reduplicated wh-words, neither of which are in subject position, but it nevertheless seems to get a 'distributive' interpretation, which was seen to be impossible with Yaeyaman reduplicated objects.

It seems that reduplicated indeterminates in Korean multiple wh-questions are always interpreted as resulting from agreement with a higher RED that requires non-atomic answers, rather than a local RED requiring non-atomic entities. If reduplication could be interpreted as requiring non-atoms in the set of entities denoted by the indeterminate, we would expect (27) to be compatible with a singlepair answer with a plural object and indirect object, contrary to the intuitions reported above. Moreover, the higher RED in Korean seems to trigger agreement with all of the indeterminates in its scope, rather than only the highest one, as in Yaeyaman. Finally, as we saw earlier, reduplication in Korean is associated with a stronger presupposition than in Yaeyaman; here, it seems that reduplication forces a pairlist answer. This contrasts with 'distributive' reduplication of Yaeyaman subject indeterminates, which simply puts plural answers into play, without excluding single-pair answers. 10

b. List Answer Bill likes Smith, Sue Jones, ...

(Chierchia 1993: 181)

The observation is that a quantifier in subject position can be distributed with respect to a wh-word in object position, resulting in a pair-list interpretation. This cannot happen with a quantifier in object position and wh-word in subject position. The parallel with the data described here is clear. In Yaeyaman, plurality on subjects can be distributed over pairs, while plurality on objects cannot. While the parallel here is suggestive, I will not pursue it any further in this paper, leaving this potential line of inquiry for future research.

10 I do not at this time have data for Yaeyaman sentences parallel to (27), with two non-subject 
Plurality and distributivity in Yaeyaman wh-questions

\section{Conclusion}

I have argued that reduplication of indeterminates in Yaeyaman is the spellout of a morpheme RED, which operates on an alternative set and generates a presupposition that this set contains non-atoms. Indeterminates themselves were argued to denote contextually-restricted sets of alternative entities, potentially containing both atomic and non-atomic entities in the sense of Link 1983/2002. When RED attaches directly to the indeterminate, it adds a presupposition that this set contain at least some non-atomic entities.

I then argued that the 'distributive' reading of reduplicated subject indeterminates in multiple-wh questions results from RED attaching at the clause level. A question denotes a set of propositions. Attachment of RED at the clause level requires that this set contain some non-atomic propositions. Atomicity in this case is defined relative to the propositional algebra built from the original set of Hamblin alternatives, following Lahiri (1991, 2002). This higher RED triggers morphological agreement on the closest indeterminate, which in the examples considered here is the subject.

Although the details of how plurality operates at the propositional level require further exploration, the analysis argued for here suggests that the plural/singular, atomic/non-atomic distinction operates at the level of propositions in a way parallel to its operation at the level of entities. I have argued that the two notions can receive parallel treatments by adopting the algebraic approach to question structure developed in Lahiri 1991, 2002. Regardless of the formal details, the Yaeyaman data show that the notion of singular and plural answers to a question is a linguistically well-motivated distinction, and that the singular-plural distinction in this domain is parallel to that seen in the domain of entities.

\section{References}

Bylinina, Lisa. 2010. Wh-reduplication in Altai. In H. Maezawa \& A. Yokogoshi (eds.), The 6th Workshop on Altaic Formal Linguistics (WAFL6) MITWPL, Cambridge: MIT.

Chierchia, Gennaro. 1993. Questions with quantifiers. Natural Language Semantics 1(2). 181-234.

Chung, Daeho. 1999. On WH-doublets in Korean. Enehak 25. 281-301.

Davis, Christopher. 2014. The role of focus particles in Wh-interrogatives: Evidence from a Southern Ryukyuan language. In Robert E. Santana-LaBarge (ed.),

indeterminates. Given the discussion here, I predict that only the structurally higher of the two indeterminates will be reduplicated in pair-list contexts with singular substitutions. I also do not have data for Korean multiple-wh questions with reduplicated subject indeterminates. I leave further investigation of these issues to future research. 
The 31st West Coast Conference on Formal Linguistics (WCCFL 31), 124-133. Somerville, MA: Cascadilla Press.

Farkas, Donka F. \& Henriëtte E. de Swart. 2010. The semantics and pragmatics of plurals. Semantics and Pragmatics 3(6). 1-54. doi:10.3765/sp.3.6.

Groenendijk, Jeroen \& Martin Stokhof. 1984. Studies in the semantics of questions and the pragmatics of answers: University of Amsterdam dissertation.

Hagstrom, Paul. 1998. Decomposing questions: MIT dissertation.

Hamblin, C.L. 1973. Questions in Montague English. Foundations of Language 10. 41-53.

Haspelmath, Martin. 1997. Indefinite Pronouns. Oxford: Oxford University Press.

Heim, Irene. 1991. Artikel und Definitheit. In Arnim von Stechow \& Dieter Wunderlich (eds.), Semantik: Ein internationales Handbuch der zeitgenössischen Forschung, 487-535. Berlin: de Gruyter.

Heinrich, Patrick, Shinsho Miyara \& Michinori Shimoji (eds.). 2015. Handbook of the Ryukyuan Languages. Berlin: de Gruyter Mouton.

Hiraiwa, Ken. 2009. Kakarimusubi in Okinawan and its microparametric implications. Snippets 19. 13-14.

Kim, Jeong-Seok. 1999. A note on multiple wh-singlet/doublet questions. Studies in Generative Grammar 9(2). 381-402.

Kratzer, Angelika \& Junko Shimoyama. 2002. Indeterminate pronouns: The view from Japanese. In Yukio Otsu (ed.), The Third Tokyo Conference on Psycholinguistics (TCP 2002), 1-25. Hituzi Syobo.

Kuroda, Sige-Yuki. 1965. Generative grammatical studies in the Japanese language: MIT dissertation.

Lahiri, Utpal. 1991. Embedded interrogatives and the predicates that embed them: MIT dissertation.

Lahiri, Utpal. 2002. Questions in Embedded Contexts. Oxford: Oxford University Press.

Lasersohn, Peter. 1998. Generalized distributivity operators. Linguistics and Philosophy 21(1). 81-93.

Link, Godehard. 1983/2002. The logical analysis of plurals and mass terms: A lattice-theoretical approach. In Paul H. Portner \& Barbara H. Partee (eds.), Formal Semantics: The Essential Readings, chap. 4. Wiley-Blackwell.

Maeara, Tooru. 2011. Taketomi Hougen Jiten [Taketomi Dialect Dictionary]. Nanzansha.

May, Robert. 1985. Logical Form: Its Structure and Derivation. Cambridge, Massachusetts: MIT Press.

Miyara, Shinsho. 2007. On the properties of Wh and focus in Okinawan. In Bjarke Frellesvig, Masayoshi Shibatani \& John Charles Smith (eds.), Current Issues in the History and Structure of Japanese, 187-205. Kurosio. 
Plurality and distributivity in Yaeyaman wh-questions

Nakanishi, Kimiko \& Satoshi Tomioka. 2004. Japanese plurals are exceptional. Journal of East Asian Linguistics 13. 113-140.

Roberts, Craige. 1987. Modal subordination, anaphora, and distributivity: University of Massachusetts Amherst dissertation.

Sauerland, Uli, Jan Anderssen \& Kazuko Yatsushiro. 2005. The plural is semantically unmarked. In Stephan Kepser \& Marga Reis (eds.), Linguistic Evidence, 413434. Berlin: Mouton de Gruyter.

Shimoji, Michinori \& Thomas Pellard (eds.). 2010. An Introduction to Ryukyuan Languages. Tokyo: ILCAA.

Shimoyama, Junko. 2001. Wh-constructions in Japanese: University of Massachusetts Amherst dissertation.

Shimoyama, Junko. 2006. Indeterminate phrase quantification in Japanese. Natural Language Semantics 14. 139-173. doi:10.1007/s11050-006-0001-5.

Shimoyama, Junko. 2011. Japanese indeterminate negative polarity items and their scope. Journal of Semantics 28(4). 413-450. doi:10.1093/jos/ffr004.

Shinzato, Rumiko \& Leon A. Serafim. 2013. Synchrony and Diachrony of Okinawan Kakari Musubi in Comparative Perspective with Premodern Japanese. Languages of Asia. Leiden: Global Oriental.

Sudo, Yasutada. 2013. Metalinguistic quantification: Evidence from Japanese wh-doublets. Ms., Institut Jean Nicod.

Sugahara, Mariko. 1996. Shuri Okinawan kakari-musubi and movement. In Masatoshi Koizumi, Masayuki Oishi \& Uli Sauerland (eds.), Formal Approaches to Japanese Linguistics (FAJL 2), 235-254. Cambridge, MA: MITWPL.

Uemura, Yukio \& Wayne P. Lawrence. 2003. The Ryukyuan Language. Endangered Languages of the Pacific Rim. Osaka, Japan: ELPR.

Christopher Davis

University of the Ryukyus

Faculty of Law and Letters

1 Senbaru, Nishihara

Okinawa, Japan 903-0213

cmdavis@11.u-ryukyu.ac.jp

cmdavis.linguist@gmail.com

http://cmdavis.org 